
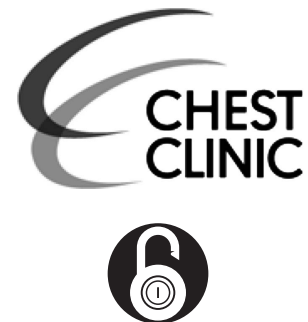

\section{OPEN ACCESS}

- Additional material is published online only. To view please visit the journal online (http://dx.doi.org/10.1136/ thoraxjnl-2014-205248).

${ }^{1}$ Hofstra North Shore LIJ School of Medicine and North Shore LIJ Health System, Department of Medicine, Manhasset, New York, USA ${ }^{2}$ Department of Population Health, New York University School of Medicine, New York, New York, USA

${ }^{3}$ Division of Pulmonary and Critical Care, University of Washington School of Medicine, Seattle, Washington, USA

\section{Correspondence to} Dr Negin Hajizadeh, 1985 Marcus Avenue, Suite LL 104, Lake Success, NY 11042, USA; NHajizadeh@nshs.edu

Received 19 February 2014 Revised 10 April 2014 Accepted 18 April 2014 Published Online First 14 May 2014

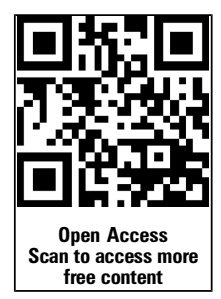

CrossMark

To cite: Hajizadeh $\mathrm{N}$, Goldfeld K, Crothers K. Thorax 2015;70:294-296.

\title{
What happens to patients with COPD with long-term oxygen treatment who receive mechanical ventilation for COPD exacerbation? A 1-year retrospective follow-up study
}

\author{
Negin Hajizadeh, ${ }^{1}$ Keith Goldfeld ${ }^{2}$ Kristina Crothers $^{3}$
}

\section{ABSTRACT}

We performed a retrospective cohort study of patients with chronic obstructive lung disease (COPD) on longterm oxygen treatment (LTOT) who received invasive mechanical ventilation for COPD exacerbation. Of the 4791 patients, $23 \%$ died in the hospital, and $45 \%$ died in the subsequent 12 months. $67 \%$ of patients were readmitted at least once in the subsequent 12 months, and $26.8 \%$ were discharged to a nursing home or skilled nursing facility within 30 days. We conclude that these patients have high mortality rates, both in-hospital and in the 12 months postdischarge. If patients survive, many will be readmitted to the hospital and discharged to nursing home. These potential outcomes may support informed critical care decision making and more preference congruent care.

\section{INTRODUCTION}

Chronic obstructive lung disease (COPD) is the third leading cause of death in the USA. The disease follows a progressive course, and patients with advanced disease most often die from respiratory failure or cardiovascular disease. Patients hospitalised for respiratory failure due to COPD (COPD exacerbation) have a 2-year median survival, and $50 \%$ of patients who survive hospitalisation are readmitted within 6 months of discharge. ${ }^{1}$ In addition, patients with COPD are more likely to die after receiving life-supporting technologies and less likely to receive palliative care compared with lung cancer patients. ${ }^{2}$ Unless patients explicitly choose to forego invasive treatments, often the default is to 'do everything' to prolong survival. However, survivors of invasive treatments often have decreased functional capacity, are discharged to a nursing home and have recurrent hospitalisations that may adversely impact quality of life.

Knowledge of these potential outcomes may influence patients' informed decision making about whether to accept invasive mechanical ventilation. Unfortunately, few studies have measured outcomes beyond hospital survival for patients with COPD.

In this study, we describe outcomes for patients with COPD on LTOT who are admitted to an ICU for respiratory failure and receive invasive mechanical ventilation. The focus of our analysis is on outcomes that may inform patient-level decision making about whether to accept invasive mechanical ventilation, including in-hospital and 12-month mortality, readmissions and discharge to nursing home. Further, we describe the association between patient-level factors such as comorbidities and nursing home residence, and these outcomes.

\section{METHODS}

We conducted a cohort study of Medicare Beneficiaries who were hospitalised for a COPD exacerbation in 2008. The analytic dataset included 2007-2009 data from the following CMS data files: the Master Beneficiary Summary File Base Segments; the Medicare Provider Analysis and Review utilisation files (MEDPAR); the Home Health, Hospice and Durable Medical Equipment (DME) utilisation files; and Minimum Data Set (MDS).

\section{Cohort selection}

We confined the cohort to beneficiaries with diagnoses of COPD, with at least two claims for LTOT, admitted to non-rural ICUs, for COPD exacerbation, treated with invasive mechanical ventilation (4791 patients) (see online supplementary figure S1).

\section{Patient characteristics}

We collected data on the patient's age, race and sex, as well as nursing home or skilled nursing facility (SNF), admission before the index admission. We derived Elixhauser comorbidity measures based on the ICD-9 codes.

\section{Patient outcomes}

Primary outcomes were (1) in-hospital and 12-month mortality, (2) all-cause readmission over 12 months and (3) discharge to nursing home or SNF within 30 days. We measured discharge to nursing home using data from MDS entry dates.

\section{Statistical analysis}

We described patient characteristics using counts and proportions for all variables, except for age, for which we reported a mean (SD) value. We used bootstrap methods to estimate 95\% CIs for the proportions.

We tested the association of individual patient-level factors on mortality (in-hospital and 12-month) and readmission (12-month) using simple logistic regression. Fully adjusted models were estimated using multiple logistic regression and a backwards elimination variable selection algorithm. ORs and 95\% CIs were 
estimated for each of the analyses. For additional description of methods, see online supplementary material.

\section{RESULTS}

The study cohort was defined as the 4791 COPD-LTOT patients who received invasive mechanical ventilation for COPD exacerbation. Mean age was 74.3 (SD 6.4), and 88.2\% were white. $14.3 \%(n=687)$ were in a nursing home or SNF within 365 days before the index admission (defined as the first ICU admission in 2008) (see online supplementary table S1). Most patients $(69.8 \%)$ had more than three comorbidities.

\section{Mortality}

Of the 4791 COPD-LTOT patients who received invasive mechanical ventilation for COPD exacerbation, $23.3 \%$ died in the hospital and $45.2 \%$ died in the 12 months after hospital discharge (figure 1 ). Of the total cohort, $31.5 \%$ were alive 1 year following discharge.

\section{Readmissions}

Of the 3677 index admission survivors, $67.1 \%$ were readmitted at least once in the subsequent 12 months, and $45.3 \%$ were rehospitalised two or more times (figure 1). Of those with at least one readmission $(n=2466), 52.1 \%$ were readmitted due to COPD.

\section{Discharge to nursing home}

Of the 3177 patients discharged alive who were not in a nursing home in the 12 months before the index admission, $26.8 \%$ were admitted to a nursing home within 30 days after hospital discharge. An additional $12.3 \%$ of survivors were admitted to a nursing home after 30 days.

\section{Factors associated with mortality}

(See online supplementary table S2). The odds of dying in the hospital increased by $4 \%$ for every 1 year increase in age (adjusted OR (AOR), 1.04; supplement, 95\% CIs). Other factors associated with hospital death included: white race (23.8\% vs $18.9 \%$; AOR 1.42); male sex (25.3\% vs $21.7 \%$; AOR 1.17); and nursing home admission at any point in previous year $(27.2 \%$ vs $22.6 \%$; AOR 1.27). The following comorbidities were associated with hospital death: weight loss
(AOR 1.30), renal failure (AOR 1.42) and metastatic cancer (AOR 1.92). Obesity was associated with reduced mortality (AOR 0.71).

Among patients discharged alive $(\mathrm{n}=3677)$, the odds of dying within 12 months increased by $1 \%$ for every 1 year increase in age (AOR $1.01 ; 95 \%$ CI 1.01 to 1.01 ). Other factors associated with dying within 12 months included white race $(50.6 \%$ vs $46.5 \%$; AOR 1.07); male sex (52.4\% vs 48.5\%; AOR 1.04 ) and nursing home admission at any point in previous year $(63.6 \%$ vs $48.0 \%$; AOR 1.10). The following comorbidities were associated with a higher likelihood of dying within 12 months: fluid and electrolyte disorders (AOR 1.05), weight loss (AOR 1.12), solid tumour without metastasis (AOR 1.20) and metastatic cancer (AOR 1.23). Obesity was associated with reduced mortality (AOR 0.91).

\section{Factors associated with readmission}

(See online supplementary table S3). The odds of readmission were increased by $14 \%$ if patients were admitted to a nursing home within 30 days of discharge from the hospital $(75.6 \%$ vs $64.5 \%$, AOR $1.14 ; 95 \%$ CI 1.10 to 1.18$)$. The following comorbidities were associated with a higher likelihood of readmission within 12 months: congestive heart failure (AOR 1.06), diabetes (AOR 1.06), deficiency anemias (AOR 1.05) and depression (AOR 1.05).

\section{DISCUSSION}

We describe outcomes over 12 months for patients with COPD on LTOT who are treated with invasive mechanical ventilation for acute COPD exacerbation. Beyond mortality, we provide insights into the clinical care trajectories for patients after mechanical ventilation in terms of readmission and nursing home admission.

Patients with advanced COPD are at increased risk for severe COPD exacerbations that require critical care. Our work may be useful to inform patients' decision making about whether to accept invasive mechanical ventilation in the event of respiratory failure due to COPD exacerbation, by describing and quantifying survival and outcomes that may affect quality of life such as rehospitalisation and admission to nursing home.

In prior work, we designed a Markov decision model to simulate outcomes for patients with severe COPD who chose

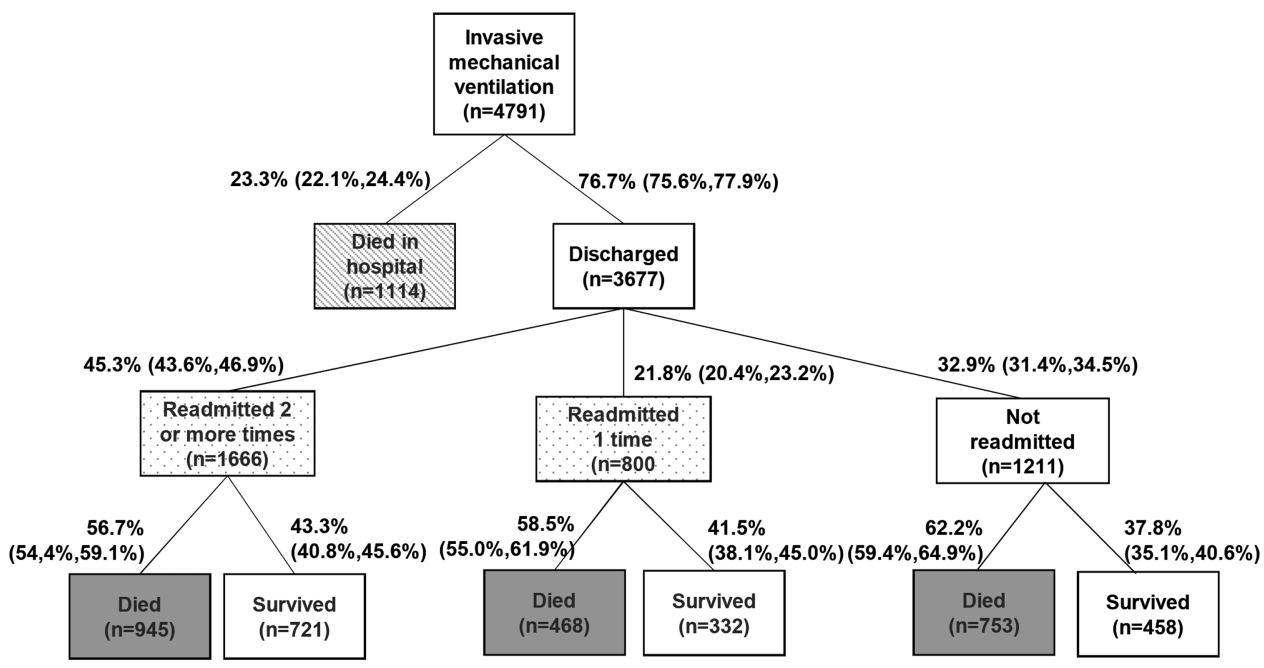

Figure 1 Outcomes over 12 months for patients with COPD-LTOT treated with invasive mechanical ventilation (with 95\% Cls). COPD-LTOT, chronic obstructive lung disease-long-term oxygen treatment. 
alternative advance directives for mechanical ventilation. We estimated 54\% 12-month mortality for severe patients with COPD who chose to receive invasive mechanical ventilation (Full Code advance directives) versus 57\% for patients who chose not to receive invasive mechanical ventilation. ${ }^{3}$ However, Full Code patients had increased rehospitalisations and discharge to nursing home. Other investigators have indicated high mortality for patients with COPD who require mechanical ventilation for COPD-related respiratory failure. Breen et al describe $48.6 \% 12$-month mortality in a retrospective cohort of 74 patients admitted to the ICU with acute respiratory failure due to COPD. ${ }^{4}$

COPD is a leading cause of hospital readmissions. Most studies report 30-day readmission because this may be more likely a marker of quality of care. The goal of our analysis was to describe outcomes that may be helpful for patients in decision making, and we therefore describe longer-term readmission rates. These readmissions likely reflect the severity of underlying chronic disease and the 'end-of-life' stage of COPD, although the proportion of potentially preventable admissions cannot be measured by this analysis.

In contrast to mortality and readmission rates, few studies have described discharge to nursing home after mechanical ventilation for COPD respiratory failure. Although our current analysis does not include data to assess functional capacity, more than $60 \%$ of nursing home residents who have COPD require extensive assistance, or are totally dependent for activities of daily living in other studies. ${ }^{5}$ In addition, many patients are unable to be liberated from mechanical ventilation ${ }^{1}$ and many of the $26.8 \%$ of patients we identified as being discharged to nursing home within 30 days may have remained on mechanical ventilation. Understanding the likelihood of nursing home admission after mechanical ventilation for COPD exacerbation, described in our study, may be valuable for patients' decision making.

Our study has several limitations. Most notably, we used LTOT as a marker of COPD severity. We chose this method as a surrogate marker of advanced/severe COPD because CMS data files do not contain lung function tests. We believe oxygen supplementation is a valid surrogate marker of COPD severity because patients with COPD typically require oxygen supplementation once their lung disease is very advanced. Second, there are limits on the generalisability of our outcomes because the cohort was predominantly white and did not include patients admitted to rural hospitals where practice patterns are likely different. Third, because this was a course-of-disease study, we did not compare outcomes from another group, such as an exacerbated COPD cohort who did not receive invasive mechanical ventilation. A meaningful comparison would require the groups to have similar characteristics (eg, health status and acuity of illness) at the time of ICU admission, which we could not ascertain with the available data.

The outcomes we report have important implications for doctor-patient discussions about prognoses in the event of acute COPD exacerbation and could be useful to patients making informed decisions about whether to accept invasive mechanical ventilation. Future work will describe the quality of life for patients discharged to a nursing home in an attempt to further inform advance care planning in patients with advanced COPD.

Contributors NH conceived and designed the study, acquired data and drafted the manuscript; NH and KF analysed and interpreted data; all authors critically revised the manuscript for important intellectual content.

Funding This work was supported in part by a grant from the U.S. Agency for Healthcare Research and Quality (K12 HS019473-01).

Competing interests None.

Ethics approval New York University School of Medicine Institutional Review Board.

Provenance and peer review Not commissioned; externally peer reviewed.

Open Access This is an Open Access article distributed in accordance with the Creative Commons Attribution Non Commercial (CC BY-NC 3.0) license, which permits others to distribute, remix, adapt, build upon this work non-commercially, and license their derivative works on different terms, provided the original work is properly cited and the use is non-commercial. See: http://creativecommons.org/ licenses/by-nc/3.0/

\section{REFERENCES}

1 Connors AF Jr, Dawson NV, Thomas C, et al. Outcomes following acute exacerbation of severe chronic obstructive lung disease. The SUPPORT investigators (Study to Understand Prognoses and Preferences for Outcomes and Risks of Treatments). Am J Respir Crit Care Med 1996;154(4 Pt 1):959-67.

2 Claessens MT, Lynn J, Zhong Z, et al. Dying with lung cancer or chronic obstructive pulmonary disease: insights from SUPPORT. Study to Understand Prognoses and Preferences for Outcomes and Risks of Treatments. J Am Geriatr Soc 2000;48(5 Suppl):S146-53.

3 Hajizadeh N, Crothers K, Braithwaite RS. Using modeling to inform patient-centered care choices at the end of life. J Comp Effectiveness Res 2013;2:497-508.

4 Breen D, Churches T, Hawker F, et al. Acute respiratory failure secondary to chronic obstructive pulmonary disease treated in the intensive care unit: a long term follow up study. Thorax 2002;57:29-33.

5 Zarowitz BJ, O'Shea T. Chronic obstructive pulmonary disease: prevalence, characteristics, and pharmacologic treatment in nursing home residents with cognitive impairment. J Manag Care Pharm 2012;18:598-606. 